\title{
Rolf Schmucker \\ Solidarität in der europäisierten \\ Gesundheitspolitik? \\ Zum Verhältnis von Wettbewerb und Solidarität im \\ europäischen Binnenmarktprojekt
}

Diskussionspapier 2008 - 2

\author{
Institut für Medizinische Soziologie \\ Fachbereich Humanmedizin der Johann Wolfgang Goethe-Universität \\ Frankfurt am Main \\ Juni 2008
}


Institut für Medizinische Soziologie

Direktor: Prof. Dr. Dr. Thomas Gerlinger

Zentrum für Gesundheitswissenschaften

Fachbereich Medizin der Johann Wolfgang Goethe-Universität

Theodor-Stern-Kai 7

60590 Frankfurt am Main

Telefon: (0 69) $6301-7610$

Fax: $\quad$ (0 69) $6301-6621$

Website: http://www.kgu.de/zgw/medsoz

ISSN 1861-5732 


\section{Zusammenfassung}

Der Prozess der europäischen Integration wirkt zunehmend auf die Gestaltung der Gesundheitssysteme der Mitgliedstaaten ein. Die von der Kommission und dem EuGH vorangetriebene Anwendung des europäischen Binnenmarkt- und Wettbewerbsrechts auf die Gesundheitspolitik hat zur Folge, dass marktlichen Steuerungsprinzipien ein Primat gegenüber staatlicher und korporatistischer Regulierung eingeräumt wird. Die gesundheitspolitische Gestaltungskompetenz liegt bei den Mitgliedstaaten, diese haben jedoch die "vier Freiheiten“ bzw. das europäische Wettbewerbsrecht zu beachten. Das Prinzip der Solidarität spielt in den europäischen Verträgen dagegen nur eine untergeordnete Rolle. Solidarität erscheint im europäischen Diskurs als ein Wert, der für die Europäische Union einen wichtigen Bezugspunkt darstellt, ohne dass er eine rechtlich verbindliche Form erhalten hat. Im Resultat entscheidet daher die Auslegung des Solidaritätsprinzips durch den Gerichtshof darüber, ob solidarische Elemente in der nationalen Gesundheitspolitik mit dem europäischen Recht vereinbar sind. Dieser Mechanismus beruht nicht auf demokratisch organisierten Meinungs- und Willensbildungsprozessen, sondern ist Gegenstand schwer prognostizierbarer richterlicher Interpretationskunst.

\section{Abstract}

European integration has a growing impact on the governance of health care systems in EU member states. The application of the European common market law to health policy, advanced by the Commission and the European Court of Justice, implies that market principles gain priority over public regulation (be it by the state or via corporatist arrangements). The competencies for shaping their health care systems lie with the member states, but their regulations have, as far as possible, to meet European market law. By contrast, the principle of solidarity is of minor importance in the European treaties. Though the notion of solidarity, in the European political discourse, is a term that constitutes a strong reference point for the European Union, it is, hitherto, by no means legally binding. As a result, it is subject to the rulings of the European Court of Justice which are, moreover, difficult to predict, and not to democratic decision-making, whether elements of solidarity in the health care sector are compatible with European law. 



\section{Inhalt}

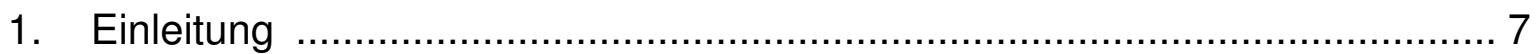

2. Solidarische Gesundheitspolitik im Wandel........................................... 7

3. Die Übertragung der europäischen Markt- und Wettbewerbsordnung auf die Gesundheitspolitik ............................................................... 12

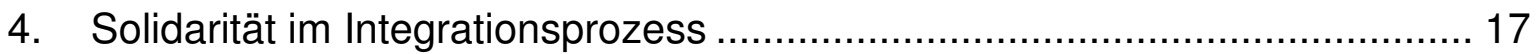

4.1. Deklamatorische Solidarität ..................................................... 18

4.2. Solidarität in den europäischen Verträgen ..................................... 20

4.3. Solidarität als Ausnahmetatbestand............................................. 23

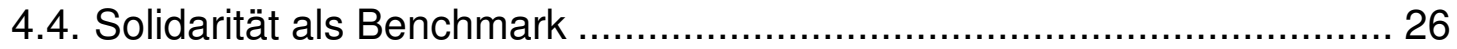

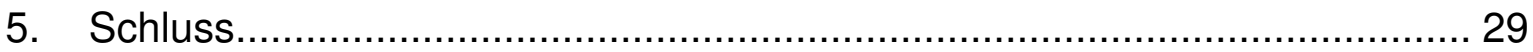

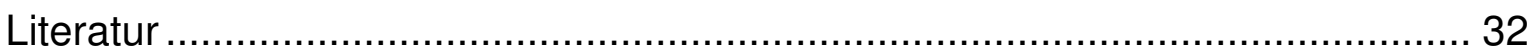





\section{Einleitung ${ }^{1}$}

Die politische Steuerung von Gesundheitspolitik geschieht zunehmend unter den Vorzeichen der europäischen Integration. Die Abgrenzung zwischen europäischer Marktintegration und nationaler Sozial- und Gesundheitspolitik ist in den vergangenen Jahren immer brüchiger geworden. Dabei spielt das europäische Markt- und Wettbewerbsrecht sowie seine (Un-)Vereinbarkeit mit den Steuerungsinstrumenten der Gesundheitssysteme der Mitgliedstaaten eine zentrale Rolle. Die große Bedeutung fiskal- und wettbewerbspolitischer Parameter im Rahmen eines sich herausbildenden europäischen Mehrebenensystem wirft die Frage nach dem künftigen Stellenwert solidarischer Prinzipien in der Gesundheitspolitik auf. Während die EU hinsichtlich des Binnenmarktes über eine stabile rechtliche und institutionelle Grundlage verfügt, mit fest verankerten Grundprinzipien und einer bindenden Rechtsprechung, bleibt die soziale Dimension auf der europäischen Ebene in einem diffusen Zustand zwischen Absichtserklärungen, einzelnen, häufig binnenmarktbezogenen Aktivitäten und nationalen Zuständigkeiten. Vor diesem Hintergrund wird im Folgenden der Frage nachgegangen, welche Bedeutung (gesundheitspolitische) Solidarität in der europäischen Markt- und Wettbewerbsordnung besitzt. Zu diesem Zweck wird der Solidaritätsbegriff in der Gesundheitspolitik diskutiert und den gesundheitspolitischen Integrationsmechanismen in der EU gegenübergestellt. Anschließend werden verschiedene Bereiche des Integrationsprozesses daraufhin analysiert, inwiefern sie auf solidarische Normen Bezug nehmen und welche Wirkungskraft diese besitzen. Unabhängig von der Diskussion, ob bzw. in welchem Umfang ein solidarischer Wettbewerb oder ein regulierter Wohlfahrtsmarkt eine Kombination von solidarischen und wettbewerblichen Steuerungsinstrumenten erlaubt, soll hier der grundsätzlichen Frage nachgegangen werden, wie das Verhältnis von Wettbewerb und Solidarität im europäischen Rahmen bestimmt ist und welche Konsequenzen dies für die Gestaltung der Gesundheitssysteme mit sich bringt.

\section{Solidarische Gesundheitspolitik im Wandel}

Solidarität ist ein schillernder Begriff. Vom fürsorgenden, barmherzigen Handeln bis zur Vorstellung einer allgemeinen „Brüderlichkeit“, von der Solidarität als „Bindemittel“ sozialer Zusammenhänge über den politischen Kampfbegriff bis hin zur Begründung wohl-

Dieser Beitrag erscheint in: Böckmann, Roman (Hrsg.)(2008): Gesundheitsversorgung zwischen Solidarität und Wettbewerb. Wiesbaden: VS Verlag. 
fahrtsstaatlicher Strukturen reichen seine moralischen, philosophischen und sozialwissenschaftlichen Konnotationen (vgl. Bayertz 1998). Als politischer Begriff, mit dem „das Bestehen einer wechselseitigen moralischen Verpflichtung zwischen Individuum und Gemeinschaft“ bezeichnet wird (ebd.: 11), erlangte „Solidarität“ seit der Französischen Revolution einen wachsenden Stellenwert. Grundsätzlich lässt sich zwischen zwei Bedeutungen unterscheiden: Zum einen wird Solidarität als moralischer Wert verstanden, der die persönlichen Einstellungen und Handlungen von Individuen prägt (das Eintreten für Arme, Schwache und Benachteiligte). Zum anderen existiert eine Vorstellung von „struktureller Solidarität“, d.h. der Unterstützung für Bedürftige, in Not geratene Personen, die nicht als mildtätige Handlung, sondern als garantiertes Recht aller Angehörigen eines Gemeinwesens konzipiert ist (Hengsbach 2001: 471). Im Folgenden wird mit Blick auf die Gesundheitspolitik vor allem die zweite Bedeutungsebene im Mittelpunkt stehen.

Strukturelle Solidarität findet ihren Ausdruck in entwickelten kapitalistischen Gesellschaften u.a. in Form wohlfahrtsstaatlicher Regulierungen, deren Grundlage in der Regel in einer verfassungsrechtlichen Kodifizierung sozialer Bürgerrechte besteht (Marshall 1992). Sie ist das institutionalisierte Resultat langwieriger sozialer Konflikte und wurde zur Legitimationsgrundlage und einem (auch ökonomisch) stabilisierenden Moment moderner Gesellschaften. Die konkreten Strukturen der Solidarität sind allerdings nicht vorgegeben, sondern das kontingente Ergebnis variierender sozioökonomischer, politischer und kultureller Entwicklungskonstellationen. Dies drückt sich u.a. darin aus, dass in ihrem Entwicklungsstand vergleichbare Gesellschaften unterschiedliche Solidaritätsstrukturen bzw. Wohlfahrtsstaatstypen ausgebildet haben (Esping-Andersen 1992). Strukturelle Solidarität ist zudem einem zeitlichen Wandel unterworfen. Veränderte gesellschaftliche Rahmenbedingungen und politische Kräfteverhältnisse führen dazu, dass überkommene Vorstellungen in Frage gestellt und konkurrierende Modelle in den Vordergrund gerückt werden, die die bestehenden Strukturen und den Bedeutungsgehalt von Solidarität verändern. Augenfälliges Beispiel für die aktuellen Umbrüche in der strukturellen Solidarität des deutschen Sozialstaats sind die im Rahmen der Agenda 2010 umgesetzten sozialpolitischen Reformen. Innerhalb des Deutungskontextes eines verschärften globalen Standortwettbewerbs erscheinen die sozialen Sicherungssysteme nun vorrangig als Kostenfaktor, der die Konkurrenzfähigkeit der inländischen Unternehmen gefährde. Zudem untergraben zu großzügige Sozialleistungen die Leistungsbereitschaft der Empfänger und seien damit für die hohe Arbeitslosigkeit mitverantwortlich. In der Konsequenz werden Reformen in der Arbeitsmarkt-, Alterssicherungs- und Gesundheitspolitik vorangetrieben, mit denen die Aus- 
gaben gesenkt und die Empfänger „aktiviert“ und zu mehr Eigenverantwortung gedrängt werden sollen (z.B. Dingeldey 2006).

Strukturelle Solidarität ist somit auch ein politisches Steuerungsinstrument, mit dem die Interaktionen und Handlungen von Individuen und Gruppen in eine gewünschte Richtung gelenkt werden sollen. Sie steht damit in Konkurrenz mit anderen Steuerungsprinzipien wie der hierarchischen oder der marktlichen Steuerung, die das Akteursverhalten über Ge- und Verbote sowie monetäre Anreize regulieren. „Im Unterschied zum marktförmigen Tausch, der gemäß dem Äquivalenzgrundsatz auf strenger, transparenter Gegenseitigkeit von Beitrag und Leistung beruht, ist die Steuerungsform der Solidarität an die Einschätzung gebunden, dass diejenigen, die sich solidarisieren, sich einander als gleich ansehen und gleich setzen, weil sie eine gleiche Interessenlage teilen, dass aber trotz oder wegen der gleichen Interessenlage ein ungleiches Verhältnis der Lebenschancen bestehen bleibt.“ (Hengsbach 2001: 472). Die Etablierung struktureller Solidarität in den Systemen der sozialen Sicherung beruhte demnach auch auf der Einsicht, dass bestimmte Güter und soziale Dienste, wenn die Verfügung über sie als soziales Bürgerrecht anerkannt wird, im Rahmen einer Markt- und Wettbewerbsordnung nicht allen Bürgern in gewünschtem Umfang zur Verfügung gestellt werden können. Als Bestandteil einer öffentlichen Daseinsvorsorge sollten sie dagegen in staatlicher Verantwortung oder durch den Staat selbst erbracht und besonderen, solidarischen Steuerungsformen unterworfen werden, die sich v.a. durch die „Nicht-Äquivalenz von Beitragszahlung und Leistungsanspruch“ auszeichnen (ebd.).

Der Bedarf an struktureller Solidarität im Gesundheitswesen wird gemeinhin mit der Besonderheit des Gutes Gesundheit begründet, die eine Verteilung über den freien Markt nicht zulasse. Die herrschende Anbieterdominanz, beruhend auf fehlender Transparenz im Leistungsgeschehen und Informationsasymmetrie zwischen Leistungserbringer und Patient, verbunden mit der vulnerablen Position des Kranken, der sich oftmals in einer Situation der Ungewissheit und Angst befindet, erschweren eine einfache Übertragung des Marktmodells auf die Gesundheitsversorgung (Deppe 2005:185ff.). Zudem lässt sich das Ziel einer umfassenden Absicherung der Bevölkerung gegen das Krankheitsrisiko schwerlich mit der Funktionsweise des privaten Versicherungsmarktes vereinbaren. Das Prinzip der Risikoäquivalenz, nach dem die Versicherungsbeiträge entsprechend des individuellen Krankheitsrisikos berechnet werden, würde - übertragen auf die gesamte Bevölkerung - dazu führen, dass eine große Gruppe (chronisch) kranker Personen ein „nicht versicherbares Risiko" darstellt und eine andere Gruppe nicht über die finanziellen Ressourcen verfügte, die notwendig wären, um eine Krankenversicherung abzuschließen. 
Das Gesundheitssystem der USA, wo etwa 45 Millionen Menschen ohne Krankenversicherungsschutz und eine noch größere Zahl ohne Krankenvollversicherung leben, verdeutlicht die möglichen Konsequenzen der Übertragung marktlicher Steuerungsmechanismen auf den Sektor der Krankenversicherung. Um solche Exklusionseffekte zu vermeiden, gibt es in einer Reihe entwickelter Wohlfahrtsstaaten umfassendere solidarische Strukturen in der Gesundheitsversorgung. Im deutschen Gesundheitswesen ist die Gesetzliche Krankenversicherung (GKV), in der knapp 90 Prozent der Bevölkerung versichert sind, eine zentrale Institution der strukturellen Solidarität. Im Sozialgesetzbuch wird die Krankenversicherung als „Solidargemeinschaft“ definiert, mit der Aufgabe, „die Gesundheit der Versicherten zu erhalten, wiederherzustellen oder ihren Gesundheitszustand zu bessern“ (§ 1 SGB V). Dabei haben die Krankenkassen „eine bedarfsgerechte und gleichmäßige, dem allgemein anerkannten Stand der medizinischen Erkenntnisse entsprechende Versorgung der Versicherten zu gewährleisten“ (§ 70 SGB V). Alle Versicherten haben, sofern dies medizinisch begründet ist, ein Recht auf eine Versorgung mit den im Leistungskatalog der GKV aufgeführten Behandlungen. Das Gesetz schreibt ein solidarisches Finanzierungsverfahren vor, in dem die Beiträge der Versicherten nicht nach Alter, Geschlecht oder Gesundheitszustand kalkuliert werden, sondern sich nach der finanziellen Leistungsfähigkeit, d.h. nach der Höhe der beitragspflichtigen Einnahmen der Mitglieder richten. Familienangehörige ohne eigenes Einkommen sind beitragsfrei mitversichert. Die „Nicht-Äquivalenz von Beitragszahlung und Leistungsanspruch“ resultiert in einem relativ hohen Grad der Umverteilung zwischen unterschiedlichen Einkommensniveaus, Geschlechtern, Familiengrößen, Altersgruppen und Gesundheitszuständen. Die solidarischen Elemente in der Finanzierungsstruktur der GKV sind eng verbunden mit einer bestimmten Gerechtigkeitsvorstellung im Gesundheitswesen. Ein wesentliches Ziel der GKV besteht in der Realisierung von Bedarfsgerechtigkeit und steht damit im Gegensatz zum Prinzip der Leistungsgerechtigkeit (,je höher die Beiträge, desto mehr Leistungen“), das eine zentrale Grundlage marktlicher Steuerungsprinzipien bildet.

Die bewusste Herausnahme gesundheitspolitischer Institutionen aus der Marktsteuerung spiegelt sich auch im besonderen kartellrechtlichen Status der GKV wider. Die Paragraphen 19 bis 21 des Gesetzes gegen Wettbewerbsbeschränkungen (v.a. die Regelungen über den Missbrauch einer marktbeherrschenden Stellung) gelten nicht für die Vertragsbeziehungen der gesetzlichen Krankenkassen mit den Leistungserbringern, sofern die Kassen oder ihre Verbände zum Abschluss der betreffenden Verträge gesetzlich verpflichtet sind ( 69 Satz 2 SGB V). Die GKV wurde vom Gesetzgeber gegenüber herkömmlichen Marktakteuren privilegiert, um die ihr zugewiesene Aufgabe - die Gesund- 
heitsversorgung der Versicherten - erfüllen zu können. Als Solidargemeinschaften, die öffentlich-rechtlich handeln, können die Kassen kein Adressat des nationalen Kartellrechts sein (Ebsen 2004). Sie sind sozusagen berechtigt, ihre „marktbeherrschende“ Stellung im Verhältnis mit den Leistungserbringern zur Geltung zu bringen. Streitigkeiten, die sich im Verhältnis der Kassen zu den Leistungserbringern ergeben, sind daher auf der nationalen Ebene sozial- und nicht kartellrechtlich zu klären. Die Festlegung von Höchstgrenzen für die Erstattung von Arzneimittelkosten (Festbeträge), mit denen die Finanzierbarkeit der Arzneimittelversorgung der Versicherten gewährleistet werden soll, ist nur ein Beispiel für den Handlungsspielraum, den die Kassen in einem nicht primär marktlichen Steuerungsumfeld besitzen.

Allerdings gilt die strukturelle Solidarität im deutschen Gesundheitswesen und in der GKV nicht uneingeschränkt. Sie findet ihre Grenzen in der parallelen Existenz von gesetzlicher und privater Krankenvollversicherung, die es einzelnen Bevölkerungsgruppen - Selbständigen, Beamten, Besserverdienenden - erlaubt, sich der solidarischen Finanzierung in der GKV zu entziehen. Etwa 10 Prozent der Bevölkerung nutzen diese Möglichkeit mit der Absicht, ihre Krankenversicherungsbeiträge zu reduzieren bzw. eine möglicherweise hochwertigere, umfangreichere oder schnellere Versorgung im Krankheitsfalle zu erhalten. Damit wird nicht nur das Solidaritätsprinzip in der Finanzierung des Gesundheitswesens geschwächt, sondern auch der Grundsatz der gleichwertigen, bedarfsgerechten Versorgung in Frage gestellt. Aber auch innerhalb der GKV stößt die Solidarität an Grenzen. Die Beitragsbemessungsgrenze bewirkt, dass Bezieher höherer Einkommen einen geringeren Anteil ihrer Einkünfte als Beitrag entrichten als die Bezieher niedriger Einkommen. Weitere Einkommensarten neben denen aus selbständiger oder abhängiger Erwerbstätigkeit wie Miet- oder Zinseinkünfte, die in den vergangen Jahrzehnten eine steigende Bedeutung erlangt haben, werden bei der Beitragsberechnung nicht mit einbezogen. Auch hier findet eine Entlastung tendenziell wohlhabenderer Bevölkerungsgruppen statt. Schließlich bewirkt die durch die Gesundheitspolitik der vergangenen Jahre betriebene Privatisierung von Krankheitskosten, z.B. durch die Erhöhung von Zuzahlungen, eine Höherbelastung von kranken Versicherte, die wiederum - sozialepidemiologisch gut belegt - überwiegend aus den niedrigeren Einkommensgruppen stammen. Entgegen den weiter oben skizzierten Prinzipien einer solidarischen, an Bedarfsgerechtigkeit orientierten Gesundheitsversorgung, tragen aktuelle Entwicklungen in der Krankenversorgungspolitik dazu bei, die soziale Ungleichheit von Zugangschancen zur gesundheitlichen Versorgung zu verstärken (Gerlinger 2007). 
Die institutionalisierte strukturelle Solidarität im deutschen Gesundheitswesen gerät zudem durch einen steuerungspolitischen Paradigmenwechsel unter Druck, der sich seit dem Gesundheitsstrukturgesetz (GSG) von 1992 abzeichnet. Die Bemühungen der Gesundheitspolitik seit den 1970er Jahren, die Ausgaben- und Beitragssatzsteigerungen in der GKV durch Kostendämpfungsgesetze zu bremsen, hatten nicht den erwünschten Erfolg gehabt. Seit Ende der 1980er Jahre wurden Elemente der Markt- bzw. Wettbewerbssteuerung in die GKV eingefügt, in der Hoffnung, dadurch eine größere Effizienz der beteiligten Akteure zu bewirken und Wirtschaftlichkeitsreserven im Gesundheitswesen zu erschließen. Auf Seiten der Krankenkassen und der Leistungserbringer werden korporatistische Arrangements der Verbände zunehmend durch einen Wettbewerb zwischen den Individualakteuren abgelöst. Neue Finanzierungsmechanismen und die Möglichkeit selektiver Vertragsgestaltung sind die Instrumente, mit denen die Akteure zu wirtschaftlichem Handeln gelenkt werden sollen. Hinsichtlich der Versicherten geht die gesundheitspolitische Tendenz in Richtung einer stärkeren Betonung der Eigenverantwortung. Solidarische und egalitäre Elemente werden zurückgedrängt (Klammer 1998). Statt dessen wird auf der Ebene des individuellen Verhaltens ein Anreizsystem geschaffen, dass die NichtInanspruchnahme von Vorsorgeleistungen durch erhöhte Zuzahlungen sanktioniert, das Selbstverschuldensprinzip in der GKV stärkt und gesunden Versicherten erlaubt, ihre solidarischen Finanzierungsbeitrag durch Selbstbehalt- und Rückerstattungstarife zu reduzieren. Es kommt zu einer Neugewichtung solidarischer und wettbewerblicher Elemente in der GKV, mit der die Tendenz zur Entsolidarisierung in der GKV verstärkt wird (Gerlinger/Mosebach/Schmucker 2007). Die Veränderungen struktureller Solidarität in der Gesundheitsversorgung gehen jedoch nicht nur auf die nationale Ebene zurück. Auch im Rahmen des europäischen Integrationsprozesses kommen verstärkt die gesundheitspolitischen Arrangements der Mitgliedstaaten auf den Prüfstand der europäischen Markt- und Wettbewerbsordnung.

\section{Die Übertragung der europäischen Markt- und Wettbewerbsordnung auf die Gesundheitspolitik}

Seit Beginn des europäischen Integrationsprozesses in den 1950er Jahren gilt eine klare Verteilung der Zuständigkeiten zwischen der europäischen Ebene und den Mitgliedstaaten. Die Kompetenzen der EG/EU liegen vorrangig auf ökonomischem Gebiet: Die Schaffung eines gemeinsamen Marktes sowie die Wirtschafts- und Währungspolitik sind ihre Kernaufgaben. Dagegen bleibt die Zuständigkeit der Mitgliedstaaten für die Gestaltung 
ihrer Sozial- und Gesundheitssysteme unberührt. Entsprechend dem Subsidiaritätsprinzip wird die Gemeinschaft in Bereichen, die nicht in ihre ausschließliche Zuständigkeit fallen, nur tätig, „,sofern und soweit die Ziele der in Betracht gezogenen Maßnahmen auf Ebene der Mitgliedstaaten nicht ausreichend erreicht werden können und daher wegen ihres Umfangs oder ihrer Wirkungen besser auf Gemeinschaftsebene erreicht werden können“ (Art. 5 EGV). Für die Gesundheitspolitik gilt, dass die Mitgliedstaaten hinsichtlich der Gestaltung, Organisation und Finanzierung der Gesundheitssysteme grundsätzlich frei sind. In Art. 152 Abs. 5 EGV wird dieses „Harmonisierungsverbot“ bekräftigt: „Bei der Tätigkeit der Gemeinschaft im Bereich der Gesundheit der Bevölkerung wird die Verantwortung der Mitgliedstaaten für die Organisation des Gesundheitswesens und die medizinische Versorgung in vollem Umfang gewahrt."

Dass es in den vergangenen Jahrzehnten dennoch zu einer schrittweisen Ausdehnung europäischer Kompetenzen auf dem Feld der Gesundheitspolitik gekommen ist, hat verschiedene Ursachen: Schon frühzeitig wurde erkannt, dass die Freizügigkeit von Arbeitskräften in der Gemeinschaft unmittelbar mit dem Geltungsbereich der Systeme der Sozialen Sicherheit kollidierte. Um die Benachteiligung von Wanderarbeitnehmern hinsichtlich ihres Sozialschutzes zu verhindern, wurde ein koordinierendes Sozialrecht geschaffen (Eichenhofer 2006). Mit der Verordnung 1408/71 (bzw. der Verordnung 883/2004) wurde geregelt, dass die Beschäftigungszeiten im europäischen Ausland so behandelt werden, als ob sie im für den Sozialschutz zuständigen Staat erbracht worden wären. Für die Krankenbehandlung bedeutete dies, dass Voraussetzungen definiert wurden, unter denen die grenzüberschreitende Inanspruchnahme medizinischer Versorgungsleistungen gewährleistet wurde (Busse/Drews/Wismar 2002: 232ff.). Die grenzüberschreitende Ausbreitung von gesundheitlichen Risiken führte dazu, dass der Gemeinschaft eine Reihe von Public Health-Kompetenzen übertragen wurden (Hervey 2002). Bestandteil der gemeinschaftlichen Aktionsprogramme sind präventive und gesundheitsfördernde Maßnahmen, die Verbesserung der Gesundheitsberichterstattung und die Abwehr grenzüberschreitender Gesundheitsrisiken, u.a. durch die Einrichtung des Europäischen Zentrums für die Prävention und die Kontrolle von Krankheiten (ECDC) in Stockholm im Jahr 2005. Auch der Verbraucherschutz und die Lebensmittelsicherheit sind gesundheitsrelevante Bereiche, in denen die EU mit Blick auf den gemeinsamen Markt eigene rechtliche und institutionelle Kapazitäten erlangte (Clergeau 2005). Im Rahmen des Binnenmarktprojektes wurde der EG zudem die Kompetenz für den Erlass von Arbeitsschutzrichtlinien übertragen. Dies geschah auch als politisches Zugeständnis an die Gewerkschaften, die im Rahmen des Binnenmarktprojektes ein verstärktes „Sozialdumping“ befürchteten. Im Er- 
gebnis wurden arbeitsplatzbezogene Sicherheits- und Gesundheitsstandards europaweit auf einem hohen Niveau harmonisiert (Gerlinger 2000). Die Vollendung des Binnenmarktes für Waren führte schließlich dazu, dass europäische Qualitäts- und Sicherheitsstandards für medizinische Produkte und Arzneimittel entwickelt wurden. Insbesondere die Regulierung der Arzneimittelsicherheit, die eine Voraussetzung für die Schaffung eines europäischen Arzneimittelmarktes darstellte, führte zu einer Kompetenzübertragung auf die europäische Ebene. Seit 1995 können Arzneimittel bei der Europäischen Arzneimittelagentur (EMEA) nach europäischen Verfahren für den gesamten Markt der EG zugelassen werden (Permanand 2006).

Die (unvollständige) Aufzählung zeigt, wie vielfältig und umfangreich die gesundheitspolitischen Kompetenzen der EG/EU mittlerweile sind. Eine strategische Rolle bei der Erweiterung der europäischen Kompetenzen spielt die Kommission, die die Dynamik der Marktschaffung ebenso zum supranationalen „Capacity-Building“ nutzte, wie das Auftreten grenzüberschreitender Gesundheitskrisen und die Interessen einflussreicher „Stakeholder" (Lamping 2007). Dennoch handelt es sich bei diesen Aspekten gesundheitspolitischer Europäisierung um intendierte Entwicklungen, die auf der Zustimmung der Mitgliedstaaten beruhen. Sie entsprechen zudem im Wesentlichen immer noch der vertraglich vorgesehenen Kompetenzverteilung: Sie ergänzen und unterstützen die Politiken der Mitgliedstaaten auf verschiedenen, definierten Feldern, berühren jedoch nicht „die Verantwortung der Mitgliedstaaten für die Organisation des Gesundheitswesens und die medizinische Versorgung“ (Art. 152 Abs. 5 EGV). Anders verhält es sich mit der Seite gesundheitspolitischer Europäisierung, die sich gegen den Willen der Mitgliedstaaten als „Spillover“ der Marktintegration entwickelt hat (Greer 2006). Das „Übergreifen“ markt- und wettbewerbspolitischer Regeln des europäischen Rechts auf die Steuerung der nationalen Gesundheitssysteme hängt mit der besonderen rechtlichen Konstruktion des Integrationsprozesses und der einflussreichen Stellung des Europäischen Gerichtshofes (EuGH) zusammen. Die oben geschilderte Kompetenzverteilung zwischen EG/EU und Mitgliedstaaten führt zu einer „konstitutionellen Asymmetrie“, in deren Rahmen weitreichende supranationale Kompetenzen mit dem Ziel der Marktöffnung bzw. der Beseitigung von Markthindernissen existieren, während der Sozialschutz lediglich eine untergeordnete Bedeutung besitzt (Scharpf 2002). Seit Mitte der 1980er Jahre wurde die Marktorientierung radikalisiert und zielt nun auf die Ausdehnung des Marktwettbewerbs in Bereiche, die vormals staatlicher bzw. öffentlicher Regulierung unterlagen. „It thus became a European governing function to eliminate national subsidies, public procurement practices and the ,privileges' of public enterprises, public utilities and public services that could be construed as 
distortions of free market competition." (Scharpf 2000: 9). In der Konsequenz stehen mitgliedstaatliche Steuerungsinstrumente im sozial- und gesundheitspolitischen Bereich unter dem Generalverdacht, die Entwicklung des Wettbewerbs und des Binnenmarktes zu behindern.

Die Europäische Kommission als „Hüterin der Verträge“ besitzt mit dem Vertragsverletzungsverfahren nach Artikel 226 EGV ein Instrument, mit dem (vermutete) Verstöße der Mitgliedstaaten gegen die vertraglichen Grundlagen geahndet werden können. Das Verfahren verläuft über verschiedene Stufen. Kommt der jeweilige Mitgliedstaat den Aufforderungen der Kommission zur Korrektur der beanstandeten Maßnahme nicht nach, kann die Kommission den Gerichtshof anrufen. Die besondere Stellung des EuGH im Integrationsprozess resultiert aus der Anerkennung von Rechtsdoktrinen durch die Mitgliedstaaten, die vertraglich nicht unmittelbar fixiert sind. In den 1960er Jahren entwickelte der EuGH zum einen die „Doktrin von der unmittelbaren Anwendbarkeit“ des EG-Rechts (1963), nach der nicht nur die Gemeinschaften und die Mitgliedstaaten betroffen werden, sondern auch subjektive Rechte begründet werden, d.h. primäres und sekundäres Gemeinschaftsrecht (Ausnahme: Richtlinien) erzeugt gerichtlich durchsetzbare Rechte auch im Verhältnis zwischen Staat und Bürgern sowie zwischen den Bürgern. Zum anderen formulierte der Gerichtshof die „Doktrin des Vorrangs des Gemeinschaftsrechts“ (1964), mit denen den Normen des EG-Rechts den Normen des nationalen Rechts übergeordnet werden. Beide Doktrinen bewirken eine „Sperrwirkung“ mit der Folge, dass ein Mitgliedstaat nicht mehr eigenmächtig handeln darf, sobald ein Regelungsfeld vom europäischen Recht erfasst wird (Haltern 2005: 401f.). Neben dem Vertragsverletzungsverfahren sind die Vorabentscheidung, bei der nationale Gerichte dem EuGH Fragen über die Auslegung des Gemeinschaftsrecht vorlegen, sowie Nichtigkeitsklagen, bei denen neben Mitgliedstaaten und EG-Organen auch natürliche und juristische Personen klagebefugt sind, wichtige Auslöser für das Tätigwerden des Gerichtshofs.

Die "Integration durch Recht“ (Joseph Weiler) hat seit den 1990er Jahren verstärkt das Feld der Gesundheitspolitik erfasst. Mit den EuGH-Entscheidungen „Kohll“ (Rs. C-158/96) und „Decker“ (Rs. C-120/95) aus dem Jahr 1998 bestätigte der Gerichtshof die Geltung des freien Waren- und Dienstleistungsverkehrs (Art.23ff., 49ff. EGV) in den Gesundheitssystemen der Mitgliedstaaten (vgl. Schmucker 2003). Die beklagten Krankenversicherungen aus den Niederlanden und Luxemburg wurden verpflichtet, ambulante Versorgungsleistungen zu erstatten, die ihre Versicherten ohne vorherige Genehmigung im EUAusland in Anspruch genommen hatten. Das Territorialitätsprinzip in der Gesundheitsversorgung wurde zu Gunsten einer weit gehenden Patientenmobilität aufgebrochen (Stein- 
meyer 1999). Im gleichen Jahr entschied der EuGH, dass die Inanspruchnahme von Geldleistungen der deutschen Pflegeversicherung nicht davon abhängig ist, dass der Versicherte sich in Deutschland aufhält (Entscheidung „Molenaar“, Rs. C-160/96). Das Pflegegeld muss an Anspruchsberechtigte in das EU-Ausland exportiert werden. In den nachfolgenden Jahren wurde eine Reihe weiterer gesundheitspolitischer Steuerungsinstrumente durch den EuGH überprüft (vgl. zum Überblick Englaender 2007). In den Rechtssachen Vanbraekel (C-368-98), Geraets-Smits und Peerbooms (C-157-99) sowie Watts (C372/04) bekräftigte der Gerichtshof, dass auch die stationäre Versorgung eine Dienstleistung nach Art. 49 EGV darstellt. Die Genehmigung dieser Dienstleistung durch einen Versicherten im EU-Ausland ist daher nur unter eng definierten Voraussetzungen zu versagen. Dies gilt auch für nationale Gesundheitsdienste wie den britischen NHS. Nach den Kohll-/Decker-Entscheidungen hatten Vertreter der deutschen GKV argumentiert, dass die Urteile nur für Systeme gelte, in denen das Kostenerstattungsprinzip angewendet wird. Die Hoffnung, aufgrund des Sachleistungsprinzips bei der Finanzierung medizinischer Leistungen von der Geltung der Dienstleistungsfreiheit ausgenommen zu sein, wurde durch die Entscheidung Müller-Fauré und van Riet (Rs. C-385/99) im Jahr 2003 durchkreuzt. Der Gerichtshof entschied, dass auch in Sachleistungssystemen Mechanismen für die nachträgliche Erstattung von Behandlungskosten vorgesehen sein müssten, um den elementaren Grundsatz des freien Dienstleistungsverkehrs zu gewährleisten.

Die gesundheitspolitischen Steuerungsinstrumente der Mitgliedstaaten werden allerdings nicht nur durch den freien Verkehr von Waren und Dienstleistungen berührt. Auch die europäische Wettbewerbsordnung entfaltete gesundheitspolitische Wirksamkeit (vgl. Bien 2004). Im Mittelpunkt steht dabei die Frage, inwiefern es sich bei Einrichtungen des Gesundheitssystems um Unternehmen oder Unternehmensverbände handelt. Wird dies bejaht - etwa im Falle der Gesetzlichen Krankenversicherung oder der Kassenärztlichen Vereinigungen - ist die Anwendung des europäischen Wettbewerbsrechts (Art. 81ff. EGV) gerechtfertigt. Die Herausnahme der GKV aus den deutschen kartellrechtlichen Bestimmungen nach § 69 SGB V spielt dann aufgrund des Vorrangs des europäischen Rechts keine Rolle mehr. Im Jahr 2004 war die Festlegung von Höchstbeträgen für die Erstattung von Arzneimitteln durch die Spitzenverbände der GKV (Festbeträge) Gegenstand einer wettbewerbsrechtlich begründeten EuGH-Entscheidung (siehe 4.3.). Derzeit sind zwei weitere Verfahren anhängig: Der private Klinikbetreiber Asklepios Kliniken $\mathrm{GmbH}$ versucht, die in Deutschland übliche Übernahme von Defiziten kommunaler Krankenhäuser durch die Öffentliche Hand als Wettbewerbsverzerrung und unvereinbar mit dem Beihilferecht nach Art. 87 EGV erklären zu lassen; und die Kommission strengt aktuell ein Ver- 
tragsverletzungsverfahren gegen die Bundesrepublik Deutschland an, weil der Abschluss von Rabattverträgen zwischen Gesetzlichen Krankenversicherungen und Arzneimittelherstellern nicht dem europäischen Vergaberecht entspreche.

Die unvollständige Aufzählung belegt, wie weit diese nicht-intendierte Form negativer Integration mittlerweile in die Gesundheitspolitik hinein wirkt. Die europäische Markt- und Wettbewerbsordnung überlagert die alleinige Zuständigkeit der Mitgliedstaaten für die Gestaltung ihrer Gesundheitssysteme. Es kommt zu einer Verengung des gesundheitspolitischen Handlungskorridors der Regierungen durch die Zielvorgaben der europäischen Verträge. Die Überprüfung, ob ein gesundheitspolitisches Vorhaben mit dem EU-Recht vereinbar ist, wird zum festen Bestandteil der Gesetzgebungsverfahren. Dabei wird eine Rechtsgrundlage zum Bezugspunkt, „die weitgehend den Idealen einer neoliberalen Wirtschaftsverfassung“ entspricht (Scharpf 2003: 224) und die Herausnahme der Gesundheitssysteme aus der Marktsteuerung in Frage stellt. Eine vollständige Umstellung der Gesundheitssysteme auf eine marktliche Steuerung wird allerdings aus den oben genannten, an solidarischen Zielen orientierten Gründen von den wenigsten Akteuren gefordert. Fraglich ist allerdings, welchen Stellenwert solidarische Prinzipien in der speziellen rechtlichen Konstruktion der EG/EU besitzen. Hier bietet sich eine Reihe von Ansatzpunkten, die im Folgenden genauer betrachtet werden sollen.

\section{Solidarität im Integrationsprozess}

Die vielfältigen Bedeutungen des Solidaritätsbegriffs finden sich auch in den Debatten um den Europäischen Integrationsprozess wieder. Insbesondere die Rede vom „Europäischen Sozialmodell“ zur Beschreibung der EG/EU bzw. ihrer Zielperspektive kommt kaum ohne den Verweis auf solidarische Prinzipien aus. Dennoch ist der Stellenwert von struktureller Solidarität im Rahmen der Marktintegration eher gering. Mechanismen der Umverteilung sind - mit Ausnahme der Sozial- und Strukturfonds - auf europäischer Ebene nicht vorgesehen. Eine Politik der Umverteilung bleibt den Mitgliedstaaten vorbehalten. Wo also findet sich „Solidarität“ im Integrationsprozess? Im Folgenden werden zur besseren Strukturierung verschiedene Begriffe und Orte von Solidarität auf der europäischen Ebene unterschieden und ihre Konsequenzen für die Gesundheitspolitik diskutiert. 


\subsection{Deklamatorische Solidarität}

Mit dem Begriff der deklamatorischen Solidarität soll der Sachverhalt beschrieben werden, dass allgemeinen solidarischen Werten und Zielbestimmungen auf einer rechtsunverbindlichen Ebene ein hoher Stellenwert eingeräumt wird. Das Bekenntnis zu solidarischen Prinzipien hat im politischen Kontext oftmals die Funktion, Zustimmung der Bevölkerung zu geplanten politischen Projekten und Maßnahmen zu generieren, unabhängig davon, wie deren solidarischer Gehalt im Einzelnen zu bewerten ist. Folglich kann deklamatorische Solidarität mit Steuerungsprinzipien einher gehen, die sich an Bedarfsgerechtigkeit und Umverteilung orientieren, sie kann aber auch dazu dienen, die Abwesenheit konkreter solidarischer Prinzipien zu kaschieren, indem auf gemeinsame Wertebestände und Verfassungstraditionen verwiesen wird. Im europäischen Kontext spielt deklamatorische Solidarität in der Debatte um ein Europäisches Sozialmodell (ESM) seit Mitte der 1980er Jahre eine wichtige Rolle. Nach der „Hinwendung zum Markt“ mit der Einheitlichen Europäische Akte im Jahr 1986 (Manow/Schäfer/Zorn 2006) wuchs die öffentliche Kritik an der fehlenden sozialen Dimension des Integrationsprozesses. Die erhoffte Entfesselung der Marktkräfte durch die Vollendung des Binnenmarktes sollte nach der Vorstellung des damaligen Kommissionspräsidenten Jacques Delors in ein ESM eingebettet sein. In Abgrenzung zum US-amerikanischen Gesellschaftsmodell sollte Europa über institutionalisierte Mechanismen des sozialen Ausgleichs verfügen, mit denen Marktprozesse in sozialpolitischer Absicht reguliert werden können: „Ich sehe meine Verantwortung darin, mich um ein gerechtes Gleichgewicht zwischen Ökonomischem und Sozialem zu kümmern und bin nicht dazu da, auf den Trümmern des Sozialen für eine starke Wirtschaft zu kämpfen.“ (Delors, zit.n. Däubler 1989: 44). Trotz solcher Ankündigungen blieb die Entwicklung aktiver europäischer Sozialpolitik - mit Ausnahme des Arbeitsschutzrechts - in den folgenden Jahren nur schwach ausgeprägt (Leibfried 2006). Trotzdem - oder gerade deshalb - ist das ESM seitdem auf der deklamatorischen Ebene ein stetiger Bezugspunkt in den Stellungnahmen der relevanten europäischen Akteure. Die Unschärfe des Begriffs - Giddens spricht von einem „Gemisch von Werten, Errungenschaften und Hoffnungen, die hinsichtlich ihrer Form und des Grades ihrer Verwirklichung in den einzelnen europäischen Staaten unterschiedlich ausfallen“ (Giddens 2006) - lässt dabei Raum für vielfältige Interpretationen.

Die europäische Kommission sieht in ihrer Sozialpolitischen Agenda den Bedarf, im Rahmen der Lissabon-Strategie die „Modernisierung und Entwicklung des europäischen Sozialmodells“ voranzutreiben (Kommission 2005). Die Konzentration der Lissabon-Strategie auf Wettbewerbsfähigkeit, Wirtschaftswachstum und Arbeitsplätze prägt die Vorstellung 
künftiger Sozialpolitik. Diese müsse einen Ansatz verfolgen, „der eine positive Interaktion der wirtschafts-, sozial- und beschäftigungspolitischen Maßnahmen“ gewährleiste. Vor dem Hintergrund tief greifender wirtschaftlicher und sozialer Veränderungen in einer globalisierten Welt müsse es darum gehen, „die Systeme der sozialen Sicherung so zu modernisieren, dass sie den aktuellen Ansprüchen unserer Gesellschaft gerecht werden, und zwar auf der Grundlage der Solidarität und unter Betonung der Rolle dieser Systeme als produktiver Faktor" (ebd.:2). Die produktivistische Einbindung von Sozialpolitik verdeutlicht, dass die Deklamation von Solidarität mit unterschiedlichen Zielen verbunden sein kann. Im Gegensatz zu den charakteristischen Merkmalen des ESM in den westeuropäischen Nachkriegsgesellschaften, das auf Marktregulierungen, Umverteilungen und universalistischen Elementen in der Sozialpolitik beruhte, liegt den aktuellen Debatten eine veränderte Vorstellung von der Rolle des Marktes und der Wohlfahrtsstaaten zu Grunde. Der „Sachzwang“ des Wettbewerbs rückt in den Vordergrund. Das Ziel des sozialen Augleichs verliert gegenüber den Merkmalen der Rekommodifizierung und Leistungsgerechtigkeit an Gewicht (Aust/Leitner/Lessenich 2002).

Die zunehmende gesundheitspolitische Relevanz des Integrationsprozesses hat im Jahr 2006 dazu geführt, dass der Europäische Rat das ESM für den Bereich der Gesundheitspolitik präzisierte. Auf der Luxemburger Ratstagung, die sich mit den Themen Beschäftigung, Sozialpolitik, Gesundheit und Verbraucherschutz befasste, wurde eine Erklärung mit dem Titel "Gemeinsame Wert und Prinzipien in den Europäischen UnionGesundheitssystemen“ verabschiedet (Rat 2006). Die Erklärung ist eine Reaktion auf die vorausgegangenen gesundheitspolitisch relevanten Entwicklungen im Rahmen des Integrationsprozesses, die aus den Wechselwirkungen zwischen der europäischen Markt- und Wettbewerbsordnung sowie den nationalen Gesundheitssystemen resultierten. Der Rat bezieht sich explizit auf die gesundheitspolitisch einschlägige Rechtsprechung des EuGH und die Absicht der Kommission, Regelungen über den Verkehr mit Gesundheitsdienstleistungen im europäischen Sekundärrecht zu verankern. In der Erklärung betont der Rat seine Überzeugung, „dass die Entwicklungen in diesem Bereich aus einem politischen Konsens und nicht nur aus der Rechtsprechung erwachsen sollten“ (ebd.: 2). Die Grundlagen eines solchen Konsenses werden in dem Ziel der Mitgliedstaaten gesehen, über ein hohes Gesundheitsschutzniveau einen Beitrag für sozialen Zusammenhalt, soziale Gerechtigkeit und nachhaltige Entwicklung zu leisten. Dabei wird eingeräumt, dass die praktische Umsetzung der gemeinsamen Werte und Prinzipien in den Mitgliedstaaten sehr unterschiedlich ausfällt. In einer kurzen Passage werden die gemeinsamen Werte be- 
nannt, die quasi eine gesundheitspolitische Zuspitzung des Europäischen Sozialmodells darstellen:

„Die Grundwerte Universalität, Zugang zu einer Gesundheitsversorgung von guter Qualität, Gleichbehandlung und Solidarität finden im Handeln der verschiedenen EU-Organe breite Zustimmung. Zusammen bilden sie ein Wertgefüge, das in ganz Europa geteilt wird. Universalität bedeutet, dass niemandem der Zugang zur Gesundheitsversorgung verwehrt ist; Solidarität ist eng verbunden mit der finanziellen Gestaltung unserer nationalen Gesundheitssysteme und dem Erfordernis, die Zugänglichkeit für alle zu gewährleisten; Gleichbehandlung bezieht sich auf gleichen Zugang je nach den Bedürfnissen, unabhängig von ethnischer Zugehörigkeit, Geschlecht, Alter, sozialem Status oder Zahlungsfähigkeit. Die EU-Gesundheitssysteme haben auch zum Ziel, entsprechend dem Anliegen der EU-Mitgliedstaaten bestehende Ungleichheiten bei der Gesundheitsversorgung zu verringern; in enger Verbindung damit steht die Arbeit in den Systemen der Mitgliedstaaten zur Verhütung von Krankheiten, unter anderem durch die Förderung einer gesunden Lebensweise." (ebd.; Herv.i.Orig., R.S.).

Die Betonung solidarischer Ziele in sozial- und gesundheitspolitischen Absichtserklärungen bleibt - im Gegensatz zu den Bestimmungen des europäischen Markt- und Wettbewerbsrechts - zunächst einmal ohne verbindliche Konsequenzen. Deklamatorische Solidarität könnte allenfalls mittelbare Wirkung entfalten, sofern sich andere Akteure (etwa der EuGH) an die Erklärungen von Rat und Kommission gebunden fühlen. Allerdings bleibt auch dann offen, mit welchem Inhalt der Begriff der Solidarität gefüllt wird. Die unterschiedlichen Auslegungen des ESM machen deutlich, dass das Verständnis von Solidarität und die sich daran möglicherweise anschließenden Strategien einem historischen Wandel unterliegen.

\subsection{Solidarität in den europäischen Verträgen}

Der Begriff der Solidarität spielt in den europäischen Verträgen keine hervorgehobene Rolle. Im EU-Vertrag wird auf die solidarische Gestaltung der „Beziehungen zwischen den Mitgliedstaaten“ (Art. 1) und auf die „gegenseitige Solidarität“ in der Außen- und Sicherheitspolitik (Art. 11 Abs. 2) verwiesen. Im EG-Vertrag erscheint der Begriff lediglich einmal, in Art. 2, wo neben anderen Zielen die Förderung der „Solidarität zwischen den Mitgliedstaaten“ als Gemeinschaftsaufgabe benannt wird. Auch die Bestimmungen zum Gesundheitsschutz enthalten keinen direkten Hinweis auf solidarische Zielsetzungen der 
Gemeinschaft. Die Absicht, „einen Beitrag zur Erreichung eines hohen Gesundheitsschutzniveaus“ zu leisten (Art. 3 Abs. 1p EGV) und darauf bei der „Festlegung und Durchführung aller Gemeinschaftspolitiken und -maßnahmen“ zu achten (Art. 152 Abs.1 EGV) lässt keinerlei Rückschlüsse auf die Art und Weise zu, wie diese Ziele erreicht werden sollen. Das Primat der Marktschaffung wird an einigen Stellen lediglich mit dem Hinweis versehen, dass die getroffenen Maßnahmen die öffentliche Gesundheit nicht gefährden bzw. ein hohes Gesundheitsschutzniveau beachten sollen. Diese Vorgabe findet sich z.B. beim Verbot mengenmäßiger Beschränkungen im Warenverkehr (Art. 30), im Rahmen der Niederlassungsfreiheit (Art. 46 abs. 1) oder bei der Angleichung der Rechtsvorschriften, die der Verwirklichung des Binnenmarktes dienen (Art. 95 Abs. 3).

Ein Verweis auf soziale Grundrechte findet sich in Art. 136 EGV, wo die Europäische Sozialcharta, die 1961 vom Europarat verabschiedet wurde, und die Gemeinschaftscharta der sozialen Grundrechte der Arbeitnehmer, die auf der Ratstagung in Straßburg 1989 verabschiedet wurde, als Bezugssysteme herangezogen werden. Während in der Sozialcharta das Recht auf Schutz der Gesundheit proklamiert wird, ohne präzisere Aussagen über die gesundheitssystemische Gestaltungsprinzipien zu treffen, fordert die Gemeinschaftscharta „zufriedenstellende Bedingungen“ für den Gesundheitsschutz und die Sicherheit am Arbeitsplatz. Aus der Sozialcharta leiten sich allerdings keine individuell einklagbaren Rechte ab. Bei Verstößen von Unterzeichnerstaaten gegen die Normen der Charta kann der Europarat in einer Resolution die betroffenen Staaten dazu auffordern, die nationale Rechtslage in Übereinstimmung mit der Sozialcharta zu bringen. Die Sozialcharta verfügt jedoch über keinerlei Sanktionsmöglichkeiten. Ähnliches gilt für die Gemeinschaftscharta, die nicht die Qualität eines verbindlichen Rechtsaktes oder eines völkerrechtlichen Vertrages besitzt. Dass die Wirkung der beiden Erklärungen dennoch über einen rein deklamatorischen Akt hinausgeht, liegt daran, dass sie als Hilfsmittel zur Auslegung des EG-Vertrags herangezogen werden. Sie können der Kommission als Rechtfertigung für geplante Richtlinien dienen. Auch der EuGH greift in seinen Entscheidungen sowohl auf die Sozialcharta als auch auf die Gemeinschaftscharta als Rechtserkenntnisquelle zurück. Da die gesundheitspolitischen Aussagen der beiden Erklärungen ausgesprochen vage bleiben, besitzt der Gerichtshof hier einen weiten Interpretationsspielraum. Die Herausbildung einer gemeinschaftlichen Grundrechtsordnung durch die Rechtsprechung des EuGH entwickelte sich seit Ende der 1960er Jahre. Seit diesem Zeitpunkt betont der Gerichtshof, dass die Beachtung der Grundrechte zu den allgemeinen Grundsätzen der Gemeinschaftsordnung gehöre. Der konkrete Gehalt wurde vom EuGH in Einzelfallentscheidungen präzisiert. Dabei legten die Richter einzelne Vertragsbestimmungen 
aus (z.B. das Diskriminierungsverbot) und entwickelten und ergänzten sie auf der Grundlage gemeinsamer Verfassungstraditionen der Mitgliedstaaten. Auf diesem Wege wurde eine Reihe von Grundrechten und von grundrechtsverwandten rechtsstaatlichen Prinzipien entwickelt. Diese Art der Grundrechtsordnung musste allerdings unvollständig bleiben, da der EuGH immer im Rahmen des jeweiligen Einzelfalls zu entscheiden hatte (vgl. Borchardt 1999).

Einen wichtigen Schritt in der europäischen Entwicklung der Grundrechte ist die nach zähen Verhandlungen im Jahr 2000 verabschiedete „Charta der Grundrechte der Europäischen Union“. Sie stellt eine politische Willenserklärung ohne rechtsverbindlichen Charakter dar, soll allerdings die Organe der Gemeinschaft und die Mitgliedstaaten binden, sofern sie das Recht der Union durchführen (Art. 51). Eine Besonderheit der Charta liegt in der vergleichsweise ausgeprägten Betonung sozialer Grundrechte. Dies beginnt damit, dass bereits in der Präambel der Wert der Solidarität neben der Menschenwürde, der Freiheit und der Gleichheit als Bestandteil des „geistig-religiösen und sittlichen Erbes“ der Union benannt wird. Dies ist in anderen internationalen Menschenrechtsdokumenten nicht der Fall. Das Kapitel 4 der Charta steht unter der Überschrift „Solidarität“ und enthält eine Reihe klassischer sozialer Grundrechte. Von gesundheitspolitischer Bedeutung ist Art. 35, in dem es heißt: „Jede Person hat das Recht auf Zugang zur Gesundheitsvorsorge und auf ärztliche Versorgung nach Maßgabe der einzelstaatlichen Rechtsvorschriften und Gepflogenheiten." In den Verhandlungen über eine europäische Verfassung war vorgesehen, die Charta in den Verfassungsvertrag zu integrieren. Nach der gescheiterten Ratifizierung einigten sich die Mitgliedstaaten im Rahmen der Verhandlungen um den Reformvertrag von Lissabon darauf, der Charta europarechtlich den gleichen Wert wie den Verträgen zu verleihen. Damit soll sie ab 2009 Rechtsverbindlichkeit in der EU erlangen. ${ }^{2}$ Die besondere Bedeutung dieses Schrittes liegt darin, dass erstmals soziale Grundrechte systematisch in das Rechtssystem der EU integriert würden. Die entsprechenden Normen besäBen damit nicht länger lediglich den Status eines allgemeinen Bekenntnisses. Wie weit sich allerdings eine - durch den EuGH zu überwachende - materiell-rechtliche Wirksamkeit entfalten könnte, ist politisch und rechtlich umstritten (Tomuschat 2004: 145).

2 Großbritannien und Polen haben sich diesem Vorgehen nicht angeschlossen und sich für ein Opt-out entschieden. 


\subsection{Solidarität als Ausnahmetatbestand}

Die Anwendung des europäischen Markt- und Wettbewerbsrechts auf die nationalen Gesundheitssysteme ist vorrangig darauf ausgerichtet, (unzulässige) Behinderungen der „vier Freiheiten“ und des Wettbewerbs zu beseitigen. Der EG-Vertrag ist in seiner derzeitigen Fassung keine Rechtsgrundlage, mit der ein Ausbau von solidarischen, marktregulierenden Steuerungsinstrumenten in der Gesundheitspolitik betrieben werden könnte. Die fehlenden Kompetenzen der europäischen Ebene für die Gestaltung der Gesundheitssysteme haben den EuGH jedoch nicht daran gehindert, die eigene Zuständigkeit für gesundheitssystemische Fragen zu reklamieren, sofern sie in einen Zusammenhang mit der europäischen Marktordnung gebracht werden können. Der Grundtenor der EuGHEntscheidungen lautet: Die Mitgliedstaaten besitzen für die Gestaltung ihrer Gesundheitssysteme zwar die alleinige Kompetenz, sie müssen dabei jedoch das europäische Marktund Wettbewerbsrecht beachten. Das Primat wettbewerblicher Steuerung auf der europäischen Ebene stellt grundsätzlich gesundheitspolitische Regelwerke auf der nationalstaatlichen Ebene in Frage, die die Allokation von Gütern und Dienstleistungen nicht über den Markt organisieren. Damit kommt es zu der widersprüchlichen Konstellation, dass sich Steuerungsinstrumente im Kontext der europäischen Wettbewerbsordnung rechtfertigen müssen, die in der Absicht etabliert worden waren, durch die Herausnahme aus dem Markt solidarische Zielvorstellungen in der Gesundheitspolitik zu realisieren. Die Öffnung der nationalen Gesundheitspolitik gegenüber dem europäischen Binnenmarkt- und Wettbewerbsrecht hat - wie weiter oben aufgeführt - zu einer Reihe von EuGHEntscheidungen geführt, in denen Regulierungen der Mitgliedstaaten als nicht gerechtfertigte Verstöße gegen das europäische Recht interpretiert wurden. Die Rechtsprechung des EuGH hat allerdings an verschiedenen Punkten Einschränkungen des Markt- und Wettbewerbsrechts für zulässig erklärt. Dabei greift der EuGH bei der Legitimierung solcher „solidarischen Ausnahmetatbestände“ partiell auf nicht rechtsverbindliche Erklärungen (wie die Sozialcharta oder die Charta der Grundrechte) sowie europäische Verfassungstraditionen zurück. In diesem Zusammenhang kommt auch der - vertraglich nicht definierte - Wert der Solidarität bei der Beurteilung gesundheitspolitischer Steuerungsinstrumente zur Geltung. Dies ist vor allem dann von Bedeutung, wenn es um die wettbewerbsrechtliche Einstufung von Einrichtungen geht, die mit Aufgaben der sozialen Sicherheit betraut sind.

Eine wegweisende Entscheidung fällte der EuGH im Jahr 1993 hinsichtlich eines Rechtsstreits zwischen zwei französischen Staatsbürgern und der französischen Krankenversicherung (Rechtssache Poucet und Pistre, C-159/91 und C-160/91). Die Kläger hatten 
argumentiert, dass die Versicherungspflicht in der Krankenversicherung nicht mit den kartellrechtlichen Vorschriften des EG-Vertrags (Art. $81 \mathrm{ff}$. EGV) vereinbar sei, da so eine nicht zu rechtfertigende marktbeherrschende Stellung begründet werde. Im Kern war die Frage zu entscheiden, ob es sich bei der Krankenversicherung um ein Unternehmen im Sinne des EG-Vertrags handele. Erst wenn dies der Fall ist, greift das Wettbewerbsrecht. In einer früheren Entscheidung (Rechtssache Höfner und Elser, C-41/90) hatte der Gerichtshof alle „Einheiten“ als Unternehmen definiert, die eine „wirtschaftliche Tätigkeit“ ausüben, unabhängig von ihrer Rechtsform und der Art ihrer Finanzierung. Damit liegt eine weit gefasste Definition des Unternehmensbegriffs vor. Allerdings, so der EuGH in der Entscheidung Poucet-Pistre, erfüllten die Krankenkassen eine Aufgabe mit „ausschließlich sozialem Charakter“, die „auf dem Grundsatz der nationalen Solidarität“ und ohne Gewinnzweck ausgeübt werde. Die Leistungen der Kassen werden „Von Gesetzes wegen“ erbracht und richten sich nicht nach der Höhe der Beiträge. Das Gericht schlussfolgert, dass es sich aufgrund dieser Merkmale nicht um eine wirtschaftliche Tätigkeit handele und die Krankenkassen daher keine Unternehmen im Sinne des europäischen Wettbewerbs darstellten (Rn. 16ff.). Hinsichtlich des Grundsatzes der Solidarität nimmt der EuGH eine Präzisierung vor, die darauf abzielt, dass der Versicherungsschutz unabhängig von der Vermögenslage des Versicherten erfolge und die Finanzierung auf einkommensabhängigen Beiträgen beruhe. Ausdrücklich wird der Aspekt der Einkommensumverteilung im Finanzierungssystem erwähnt, um den solidarischen Charakter der Krankenversicherung zu kennzeichnen. Zudem existiere ein Finanzausgleich zwischen den einzelnen Systemen der sozialen Sicherheit, der ebenfalls durch solidarische Erwägungen begründet sei. Folglich stelle die Versicherungspflicht im System der Krankenversicherung in Frankreich keinen Verstoß gegen das Wettbewerbsrecht dar.

Für die deutsche GKV und ihre Spitzenverbände wurde die Frage, ob es sich bei innen um Unternehmen bzw. Unternehmensverbände handelt, zu Beginn des Jahrtausends aufgeworfen. Verschiedene Pharmahersteller hatten gegen die Festsetzung von Höchstbeträgen für die Kostenübernahme bestimmter Arzneimittel geklagt, die sich nach ihrer Auffassung nicht mit dem europäischen Wettbewerbsrecht vertrage. Der EuGH entschied unter Bezugnahme auf seine im Poucet-Pistre-Urteil vorgenommene Definition, dass es sich auch bei den deutschen Krankenkassen nicht um Unternehmen handele, da sie eine „rein soziale Aufgabe“ wahrnehmen, die auf dem Grundsatz der Solidarität beruht und ohne Gewinnerzielungsabsicht ausgeführt wird (Rn. 51ff.). Im Rahmen ihrer sozialen Aufgabe sei die Bestimmung von Festbeträgen für Arzneimittel keine wirtschaftliche Tätigkeit und unterliege daher nicht dem europäischen Kartellrecht (Rechtssachen C-264/01, C- 
306/01, C-354/01, C-355/01). Bei dieser Entscheidung handelt es sich allerdings nicht um eine unstrittige Position. Die im Rahmen der Verhandlungen des EuGH vor den Beratungen des Gerichtshof abgegebenen Schlussanträge des europäischen Generalanwalts, an dessen Empfehlungen sich der Gerichtshof in den meisten Entscheidungen orientiert, weicht von dieser Auffassung ab. Der Generalanwalt versteht die Tätigkeit der Krankenkassen als wirtschaftlich, da es ein gewisses Maß an Wettbewerb zwischen GKV-Kassen sowie mit den privaten Krankenversicherungen gebe. Da die Aufgabe, die die GKV wahrnehme, auch von privaten Unternehmen mit Gewinnerzielungsabsicht ausgeübt werden könne, sei das Wettbewerbsrecht der Gemeinschaft anwendbar. Die Spitzenverbände handelten bei der Festlegung der Höchsterstattungsbeträge als Unternehmensvereinigungen mit Eigeninteressen, die durch eine faktische Festlegung von Preisen ausdrücklich ein wettbewerbswidriges Verhalten nach den Bestimmungen des EG-Vertrags an den Tag legten. Offen ließ der Generalanwalt, ob sich dieses Verhalten rechtfertigen lasse, weil es sich beim Kassenhandeln um die Erbringung von Dienstleistungen von allgemeinem Interesse handele (Jacobs 2003).

Die Rechtsprechung des EuGH zum Unternehmenscharakter von Krankenkassen verdeutlicht den ungeklärten und widersprüchlichen Stellenwert des Solidaritätsprinzips im Rahmen der europäischen Wettbewerbsordnung. Bemerkenswert ist, erstens, dass der EuGH auch ohne explizite vertragliche Grundlage das Prinzip der Solidarität bei der Entscheidung über gesundheitspolitische Steuerungsinstrumente heranzieht und es zu einem wettbewerbsrechtlichen Ausnahmetatbestand entwickelt hat. Zweitens wird angesichts des Plädoyers des Generalanwalts deutlich, dass es sich bei der Charakterisierung der Gesetzlichen Krankenkassen als soziale Organisationen ohne Unternehmenscharakter um eine rechtlich umstrittene Position handelt. Drittens schließlich macht die Rechtsprechung des EuGH darauf aufmerksam, dass die Krankenkassen durchaus als Unternehmen charakterisiert werden können, sobald ihr Finanzierungssystem, die Prinzipien der Leistungserbringung oder ihre Wettbewerbsposition gegenüber privaten Versicherungen verändert werden. Insofern kommt nationalen Gesundheitsreformen, in denen das Steuerungsinstrument des Wettbewerbs und die erweiterten Wahlmöglichkeiten der verschiedenen Akteure eine Schlüsselrolle für die weitere Entwicklung der GKV spielen, für die europarechtliche Positionierung und die Anwendung des Wettbewerbsrechts eine entscheidende Bedeutung zu. 


\subsection{Solidarität als Benchmark}

Mit der Offenen Methode der Koordinierung (OMK) wurde seit Ende der 1990er Jahre ein neues Politikinstrument auf der europäischen Ebene entwickelt, das als „weicher" Steuerungsmechanismus gilt. Im Rahmen der Lissabon-Strategie wird die OMK als Methode europäischer Politikentwicklung für verschiedene Politikfelder vereinbart. Im Weißbuch „Europäisches Regieren“ (Kommission 2001a) werden die Grundzüge der OMK dargelegt. Mit dem Verfahren soll jenseits der Harmonisierung von Rechtsvorschriften die Kooperation und der Informationsaustausch hinsichtlich ausgewählter politischer Ziele zwischen den Mitgliedstaaten intensiviert werden. Mit der Festlegung gemeinsamer Ziele und entsprechender Indikatoren, anhand derer die Zielerreichung gemessen werden kann, sollen erfolgreiche Strategien identifiziert und somit ein politischer Lernprozess zwischen den Regierungen in Gang gesetzt werden. Auf der Grundlage einer jährlichen Berichtspflicht sollen die Fortschritte der einzelnen Mitgliedstaaten transparent gemacht werden. Diese Form des politischen Benchmarkings zeichnet sich dadurch aus, dass sie für die Mitgliedsländer keine formal bindenden Konsequenzen nach sich zieht.

Nachdem die OMK in den Bereichen Beschäftigungspolitik, Alterssicherung und soziale Inklusion bereits eingesetzt wird, wird ihre Anwendung auf das Feld der Gesundheitspolitik und Langzeitpflege seit dem Jahr 2000 schrittweise vorbereitet. Ein wesentliches Problem stellt dabei die Auswahl und weitere Entwicklung von gemeinsamen Zielen und insbesondere der entsprechenden überprüfbaren Indikatoren dar. Auf der Ebene der Zielsetzungen nahm der Europäische Rat einen Vorschlag der Kommission auf, die drei übergeordnete, langfristige Ziele für die Gesundheitspolitik der Mitgliedstaaten formuliert hatte (Kommission 2001b). Erstens: Die Mitgliedstaaten sollen allen EU-Bürgern einen Zugang zur Gesundheitsversorgung gewährleisten. Unter Verweis auf die Charta der Grundrechte der EU wird die medizinische Versorgung als ein Grundrecht anerkannt. Eine besondere Stellung im Rahmen dieses Ziels nimmt die soziale Ungleichheit und die daraus resultierenden Unterschiede im Gesundheitszustand und im Zugang zur Versorgung ein. Mit dem Ziel der Verringerung gesundheitlicher Ungleichheit ist ein wesentliches Merkmal solidarischer Gesundheitspolitik in die OMK integriert worden. Zweitens: Die Gesundheitssysteme sollen qualitativ hochwertige Leistungen anbieten. Damit soll nicht nur die öffentliche Gesundheit verbessert, sondern auch die Effizienz der eingesetzten (öffentlichen) Mittel erhöht werden. Nicht zuletzt mit Blick auf den entstehenden Binnenmarkt für Gesundheitsdienstleistungen misst die Kommission der Transparenz und der Verbesserung qualitativer Standards einen hohen Stellenwert zu. Drittens: Gesundheitspolitik soll dazu beitragen, die langfristige Finanzierbarkeit der Versorgungssysteme zu gewährleisten. Der 
angesichts des demographischen Alterns der Bevölkerungen und der Innovationen in Medizintechnik und Arzneimittelforschung zu erwartende Anstieg der öffentlichen Gesundheitsausgaben kann nach Auffassung der Kommission „langfristig untragbar" werden. Es stellt sich daher die Frage, wie strukturelle Einsparungen und Kostenkontrollen in den nationalen Gesundheitssystemen realisiert werden können. Die OMK soll auch auf diesem Gebiet zur Identifizierung von „best practices“ beitragen.

Die Präzisierung der drei Oberziele in mess- und vergleichbaren Indikatoren wirft allerdings verschiedene Probleme auf. Zum einen fehlten bislang die statistischen Grundlagen, die einen Vergleich von mittlerweile 27 Mitgliedstaaten erlauben. Die Unterschiede in den Organisationsstrukturen und Finanzierungssystemen sowie den Systemen und Methoden der Gesundheitsberichterstattung der einzelnen Länder schränken die Vergleichbarkeit erheblich ein (Schneider 2002). Zum anderen besteht ein Zielkonflikt zwischen der Absicht, bei Reduzierung sozial bedingter gesundheitlicher Ungleichheiten einen umfassenden Zugang der Bevölkerung zur Gesundheitsversorgung zu gewährleisten, und dem Vorhaben, die öffentlichen Gesundheitsausgaben zu kontrollieren, um mit einer geringen Steuer- und Abgabelast die Wettbewerbsfähigkeit europäischer Unternehmen zu unterstützen. In der Debatte um die OMK ist daher - auch aus dem deutschen Gesundheitsministerium - die Befürchtung geäußert worden, dass im Rahmen des europäischen Benchmarkings Gesundheitsausgaben primär als Kostenfaktor gesehen und gesundheitsbezogene und v.a. präventive Politikansätze „zugunsten einer rein fiskalpolitischen Betrachtungsweise verloren gehen bzw. vernachlässigt werden“ (Schreiber 2005: 152). Die Auswahl der Vergleichsindikatoren ist somit nicht allein eine Herausforderung für die Gesundheitsstatistik und -berichterstattung, sondern zudem eine politisch relevante Entscheidung, die die spätere Bewertung gesundheitspolitischer Strategien präjudiziert. Die Entwicklung der Indikatoren ist in den vergangenen Jahren auf der europäischen Ebene vorangetrieben worden. Mittlerweile liegt ein vorläufiges europäisches Indikatorentableau vor, mit dem die Entwicklung in den verschiedenen Zieldimensionen abgebildet werden soll (European Commission 2006). Allerdings bleibt das Problem vergleichbarer Daten bestehen. Für das Ziel der Qualitätsförderung wurde bislang nur ein vergleichbarer EUIndikator (die Impfrate bei Kindern) definiert. Der Zugang und die Ungleichheit der Versorgung sollen vorrangig über die Indikatoren Säuglingssterblichkeit und Lebenserwartung abgebildet werden, während es für den Umfang des Krankenversicherungsschutzes aufgrund der organisatorischen Unterschiede der einzelnen Systeme nur nationale Indikatoren gibt. Die meisten EU-Indikatoren liegen bislang für den Bereich der Nachhaltigkeit der Finanzierung vor, wo die Gesundheitsausgaben pro Kopf, die Gesundheitsausgabenquo- 
te, die Quote der öffentlichen Gesundheitsausgaben und der Anteil der Selbstzahlung an den Gesundheitsausgaben vergleichbar gemacht worden sind (vgl. Schneider et.al. 2007).

Der erste gemeinsame OMK-Bericht zur den Feldern sozialer Inklusion, Alterssicherung sowie Gesundheitsversorgung und Langzeitpflege, der auf den nationalen Strategieberichten aller Mitgliedstaaten beruht, erschien im Jahr 2007. Neben den gemeinsamen Herausforderungen, die in den drei Oberzielen benannt werden, wird darauf aufmerksam gemacht, dass die konkreten sozioökonomischen und gesundheitssystemischen Verhältnisse große Unterschiede zwischen den Mitgliedstaaten aufweisen. Hinsichtlich der Vereinbarkeit der solidarischen Zielsetzung (access) und einer nachhaltigen Finanzierbarkeit (sustainability) weist der Bericht auf den bestehenden Zielkonflikt hin. „Solidarity and equitable financing are principles inherent in the systems and all countries pledge universal rights to access. However, these do not necessarily translate into universal access and significant inequities remain. All countries are firmly committed to ensuring access to adequate health care and long-term care for everyone and refuse any trade-off between access and sustainability." (European Commission 2007: 110). In seinen Empfehlungen hinsichtlich der "best practices" ist dieser erste Bericht relativ zurückhaltend. Dies mag auch an der weiterhin unbefriedigenden Datenlage liegen. Dennoch werden einige Maßnahmen aufgelistet, die für einen beispielhaften Umgang mit dem SolidaritätsNachhaltigkeits-Konflikt stehen sollen: so etwa die Kombination von privaten Zuzahlungen mit einer Belastungs-Obergrenze in Belgien, der kostenfreie Zugang zu bestimmten ambulanten Einrichtungen für Personen unterhalb einer Einkommensgrenze in Frankreich und Österreich oder die Einführung einer Telefonberatung durch einen Allgemeinmediziner in Fällen, in denen keine andere Behandlung zugänglich ist, in Estland. Als ein „best practice example" für eine bessere Kostenkontrolle in Deutschland wird die Einführung von Fallpauschalen (DRGs) in die stationären Vergütungssysteme aufgeführt (ebd. 112).

Eine abschließende Bewertung des solidaritätsstärkenden Potenzials der OMK ist angesichts des frühen Stadiums dieses Politikprozesses nicht möglich. Es lassen sich jedoch einige begründete Vermutungen über die möglichen Effekte der OMK im Gesundheitswesen benennen. Zum einen ist der Erfolg dieser „weichen“ Steuerungsform von der Bereitschaft der Mitgliedstaaten abhängig, die Methode im beabsichtigten Sinne zu nutzen. Andernfalls werden die OMK-Berichte wenig aussagekräftig und politisch wirkungslos bleiben. Im Interesse einer Stärkung solidarischer Elemente in der Gesundheitspolitik bietet die OMK zumindest theoretisch neue Optionen. Solidarität als Benchmark - sofern geeignete Indikatoren entwickelt werden - stellt eine neue Facette in der gesundheitspoliti- 
schen Governance dar. Sie steht allerdings in einem Zielkonflikt mit der angestrebten finanziellen Nachhaltigkeit. Die Einbindung der OMK in die Lissabon-Strategie, d.h. in einen fiskal-, wettbewerbs- und beschäftigungspolitischen Kontext, sprechen dafür, dass letztlich die Kostenperspektive das angestrebte Policy Learning dominieren wird (Gerlinger/Urban 2004). Eine mögliche solidaritätsstärkende Wirkung der OMK müsste zudem dem Vorrang des europäischen Markt- und Wettbewerbsrechts genügen. Was auch immer an „best practice“-Beispielen benannt wird - die entsprechenden politischen Strategien müssten vor dem EuGH Bestand haben. "Even when responding to OMC guidelines, therefore, Member States continue to operate under exactly the same legal and economic constraints of economic integration which limit their policy choices when they are acting individually." (Scharpf 2002: 655).

\section{Schluss}

Wettbewerb und Solidarität befinden sich im Prozess der europäischen Integration in einem asymmetrischen Verhältnis. Zwar lässt sich in verschiedenen inhaltlichen, rechtlichen und diskursiven europäischen Kontexten eine Bezugnahme auf solidarische Normen finden, ihre Verpflichtungsfähigkeit ist jedoch weitaus geringer als die der existierenden markt- und wettbewerbspolitischen Instrumente. Solidarität ist in Europa vorrangig in „weichen“ Steuerungskontexten vorzufinden - übereinstimmend mit der allgemeinen Beobachtung, dass „Präambeln (...) ein bevorzugter Ort von Solidaritätsbekundungen“ sind (Denninger 1998: 320). Die „harten“ Steuerungsinstrumente liegen im Gebiet der Markschaffung und haben insbesondere in den vergangenen zehn Jahren auch eine gesundheitspolitische Wirksamkeit entfaltet. Die grundlegende Tendenz, die aus der konstitutionellen Asymmetrie resultiert, ist die Infragestellung nationaler gesundheitspolitischer Arrangements auf der Basis europäischen Rechts. Politische Steuerung über Formen der strukturellen Solidarität müssen sich im europäischen Kontext legitimieren, ohne dass es dafür eine differenzierte rechtliche Basis gibt. Es kommt zu einer Verschiebung der „Demarkationslinie“ zwischen den Aufgaben des Wohlfahrtsstaates und denen privater bzw. marktlicher Versorgung (Scharpf 2002: 650). Allerdings gibt es Hinweise darauf, dass die „weichen“ Solidaritäts-Diskurse nicht völlig wirkungslos bleiben. Immerhin wurde die bislang „härteste“ Definition solidarischer Prinzipien vom EuGH vorgenommen, der in einer wettbewerbsrechtlichen Entscheidung die Existenz solidarischer Prinzipien und Einrichtungen in den gesundheitlichen Versorgungssystemen rechtfertigte. Diese Entscheidung ließ sich nur mittels des richterrechtlichen Rückgriffs auf gemeinsame Verfassungsbe- 
stände und die Traditionen eines Europäischen Sozialmodells begründen. Die gerichtliche Auslegung dessen, was als solidarisch gilt und vom Wettbewerbsrecht auszunehmen ist, entbehrt allerdings einer demokratischen Grundlage im Sinne eines europäischen Meinungs- und Willensbildungsprozesses. So bleibt die Legitimation struktureller Solidarität im Integrationsprozess der Interpretationskunst des europäischen Gerichtshofs überlassen, der letztlich immer auf der Grundlage des Markt- und Wettbewerbsrechts zu entscheiden hat.

Die Asymmetrie im Verhältnis von Wirtschafts- und Sozialintegration bedeutet für die Mitgliedstaaten der EU, dass sie bei gesundheitspolitischen Entscheidungen die Regeln des europäischen Binnenmarktes berücksichtigen müssen. Der Handlungskorridor nationaler Gesundheitspolitik wird in Richtung marktlicher und wettbewerblicher Steuerungsoptionen verengt. Die Regierungen der Mitgliedstaaten haben mittlerweile auf diese Entwicklung reagiert, indem sie vor der Verabschiedung gesundheitspolitischer Reformen Expertisen über die Vereinbarkeit mit dem europäischen Recht einholen. Besonders drastisch ist das Beispiel der niederländischen Regierung, die im Vorfeld der großen Gesundheitsreform von 2006 direkt bei der Europäischen Kommission anfragte, ob die geplanten Veränderungen europarechtlich Bestand haben würden. Potenzielle Verstöße gegen europäisches Recht bergen die Gefahr, dass die in Frage stehende Regelung vor dem EuGH verworfen wird. Vor diesem Hintergrund kommt es zu einer Anpassung des nationalen Sozialrechts an die Rechtsprechung des EuGH. In Deutschland ist dies z.B. als Reaktion auf die Entscheidungen zu Auslandsbehandlungen im ambulanten und stationären Sektor erfolgt. Die vom EuGH entwickelten Leitsätze wurden mit dem Gesundheitssystemmodernisierungsgesetz von 2004 in das Sozialgesetzbuch übernommen. Im § 13 Abs. 4 SGB V wird das Prinzip der Kostenerstattung für ambulante Auslandsbehandlungen eingeführt, die ohne vorherige Zustimmung durch die Krankenkassen in Anspruch genommen werden. Eine Bewertung dieser Veränderung unter Berücksichtigung solidarischer Aspekte fällt widersprüchlich aus. Zwar werden Patientenrechte erweitert und die Mobilität erhöht; diese Verbesserungen, die für einen relativ kleinen Teil des gesamten Behandlungsbedarfs der GKV wirksam werden, gehen allerdings mit einer sozialrechtlichen Aufwertung des Kostenerstattungsprinzips einher. Es kommt zu einer europarechtlich induzierten Schwächung des Sachleistungsprinzips in der GKV, dessen Entstehung u.a. mit dem solidarisch motivierten Interesse begründet war, eine unmittelbare medizinische Versorgung von Menschen zu gewährleisten, die nicht über ausreichende finanzielle Mittel verfügen. Es ist davon auszugehen, dass das Kostenerstattungsprinzip eine Option darstellt, deren Vorteile v.a. von materiell besser gestellten Schichten genutzt werden können. 
Es wäre jedoch zu einfach, in erster Linie den europäischen Integrationsprozess für Vermarktlichungs- und Privatisierungstendenzen in der Gesundheitspolitik verantwortlich zu machen. Der Einsatz wettbewerblicher Instrumente ist in der deutschen Gesundheitspolitik seit Beginn der 1990er Jahre in der Hoffnung forciert worden, Effizienzsteigerungen und Kostendämpfungen realisieren zu können. Die verstärkte Nutzung wettbewerblicher Steuerungsinstrumente führt in der skizzierten europarechtlichen Konstellation allerdings dazu, dass strukturelle Solidarität begründungsbedürftig wird. Je größer die Bedeutung von Markt und Wettbewerb im Gesundheitswesen wird, desto schwieriger wird es, Ausnahmen zu legitimieren. Abweichungen von solidarischen Prinzipien, wie sie etwa im Ausbau von Selbstbehalt- und Rückerstattungstarifen in der Gesundheitsreform 2007 vorgenommen wurden (Gerlinger/Mosebach/Schmucker 2007), werfen die Frage nach den Unternehmenseigenschaften der gesetzlichen Krankenkassen neu auf. Die „Verdünnung des Solidarprinzips“ durch das GKV-WSG lässt es als fraglich erscheinen, „ob die gesetzlichen Krankenkassen nach wie vor durch Verneinung ihrer Unternehmenseigenschaft dem Einfluss des europäischen Kartellrechts entzogen werden können“ (Kingreen 2007: 48). Befürworter marktlicher Steuerung in der Gesundheitspolitik sehen daher in der konstitutionellen Asymmetrie des Integrationsprozesses eine Chance, Veränderungen durchzusetzen, die im nationalen Rahmen auf größeren Widerstand stoßen würden. Die Erkenntnis, „dass das europäische Gemeinschaftsrecht ein größeres Potential an marktwirtschaftlichen Kräften enthält als das deutsche Grundgesetz und die Verfassungen anderer Mitgliedsländer“ (Henke 2005: 146), eröffnet neue Möglichkeiten der Marktschaffung im Gesundheitswesen. Insofern stehen nationale Gesundheitspolitik und das europäische Recht in einem Wechselspiel, in dem überkommene Bestände struktureller Solidarität auch weiterhin in Frage gestellt werden. Dieser Mechanismus wird so lange wirksam sein, solange es nicht gelingt, den erodierenden nationalstaatlichen Grundlagen von Solidarität durch eine Transnationalisierung (struktureller) Solidarität im europäischen Rahmen zu begegnen (vgl. hierzu die Beiträge in Beckert u.a. 2004). 


\section{Literatur}

Aust, Andreas / Leitner, Sigrid / Lessenich, Stephan (2002), Konjunktur und Krise des Europäischen Sozialmodells. Ein Beitrag zur politischen Präexplantationsdiagnostik, in: Politische Vierteljahresschrift, 43. Jg., H. 2, S. 272-301.

Bayertz, Kurt (1998): Begriff und Problem der Solidarität. In: Ders. (Hrsg), S. 11-53.

Bayertz, Kurt (1998a): Solidarität. Begriff und Problem. Frankfurt: Suhrkamp

Beckert, Jens / Eckert, Julia / Kohli, Martin / Streeck, Wolfgang (Hrsg.): Transnationale Solidarität. Chancen und Grenzen. Frankfurt/New York: Campus.

Bien, Annika S. (2004): Die Einflüsse des europäischen Kartellrechts auf das nationale Gesundheitswesen. Berlin: Erich Schmidt Verlag.

Borchardt, Klaus-Dieter (1999): Das ABC des Gemeinschaftsrechts. http://ec.europa.eu/publications/booklets/eu_documentation/02/txt_de.pdf (Zugriff am 15.01.2008).

Busse, Reinhard / Drews, Markus / Wismar, Matthias (2002). Consumer choice of healthcare services across borders. In: Busse, Reinhard / Wismar, Matthias / Berman, Philip C. (eds.):The European Union and Health Services. The Impact of the Single European Markt on Member States. Amsterdam: IOS Press., 231-248.

Clergeau, Christophe (2005): European food safety policies: between a single market and a political crisis. In: Steffen, Monika (ed.): Health Governance in Europa. Issues, challenges and theories. London/New York: Routledge, pp. 113-133.

Däubler, Wolfgang (1989): Sozialstaat EG? Notwendigkeit und Inhalte einer Europäischen Grundrechtsakte. In: Ders. (Hrsg.): Sozialstaat EG? Die andere Dimension des Binnenmarktes. Gütersloh: Bertelsmann.

Denninger, Erhard (1998): Verfassungsrecht und Solidarität. In. Bayertz (Hrsg.), S. 319-344.

Deppe, Hans-Ulrich (2005): Zur sozialen Anatomie des Gesundheitssystems. Neoliberalismus und Gesundheitspolitik in Deutschland. Frankfurt: VAS.

Dingeldey, Irene (2006): Aktivierender Wohlfahrtsstaat und sozialpolitische Steuerung. In: Aus Politik und Zeitgeschichte 8-9/2006, S. 3-9.

Ebsen, Ingwer (2004): Kartell- und vergaberechtliche Aspekte des vertraglichen Handelns der Krankenkassen. In: Die Krankenversicherung, April, S. 95-100.

Eichenhofer, Eberhard (2006): Sozialrecht der Europäischen Union. Berlin: Erich Schmidt.

Englaender, Anna (2007). EuGH-Urteile zur grenzüberschreitenden Inanspruchnahme von Gesundheitsdienstleistungen. Observatorium für die Entwicklung der sozialen Dienste in Europa. http://www.soziale-dienste-ineuropa.de/dokumente/Aktuelles/EUGH_Urteile_Zusammenfassung.pdf (Zugriff am 15.01.2008).

Esping-Andersen, Gøsta (1990): The Three Worlds of Welfare Capitalism. Cambridge: Polity Press.

Europäischer Rat (2006). Schlussfolgerungen des Rates zum Thema „Gemeinsame Werte und Prinzipien in den europäischen Gesundheitssystemen“. In: Amtsblatt der Europäischen Union C 146 vom 22.06.2006, 1-3.

European Commission (2006): Portfolio of overarching Indicators and streamlined Social Inclusion, Pensions, and Health Portfolios. D(2006), Brussels, 7 June 2006. http://ec.europa.eu/employment_social/social_inclusion/docs/2006/indicators_en.pdf (Zugriff am 15.01.2008)

European Commission (2007): Joint Report on Social Protection and Social Inclusion [2007]. Social inclusion, Pensions, Healthcare and Long Term care. Luxemburg. 
Gerlinger, Thomas (2000): Arbeitsschutz und europäische Integration. Europäische Arbeitsschutzrichtlinien und nationalstaatliche Arbeitsschutzpolitik in Großbritannien und Deutschland, Opladen: Leske+Budrich.

Gerlinger, Thomas (2007): Soziale Ungleichheit von Gesundheitschancen: Anmerkungen zum Beitrag der Gesundheitspolitik. Diskussionspapier 2007-2. Institut für Medizinische Soziologie an der Johann Wolfgang Goethe-Universität Frankfurt a.M.

Gerlinger, Thomas / Mosebach, Kai / Schmucker, Rolf (2007): Wettbewerbssteuerung im GKVWSG. Eine Einschätzung möglicher Effekte auf das Akteurshandeln im Gesundheitssystem. In: Jahrbuch für Kritische Medizin 44. Hamburg: Argument, S. 6-24.

Gerlinger, Thomas / Urban, Hans-Jürgen (2004): Auf neuen Wegen zu neuen Zielen? Die Offene Methode der Koordinierung und die Zukunft der Gesundheitspolitik in Europa, in: Kaelble, Hartmut / Schmid, Günther (Hrsg), Das europäische Sozialmodell. Auf dem Weg zum transnationalen Sozialstaat. Berlin: edition sigma, S. 263-288.

Giddens, Anthony (2006): Die Zukunft des europäischen Sozialmodells. In: Berliner Republik, 1/2006, S. 20-29.

Greer, Scott L. (2006): Uninvited Europeanization: neofunctionalism and the EU in health policy. In: Journal of European Public Policy, 13:1, 134-152.

Haltern, Ulrich (2005), Integration durch Recht, in: Bieling, Hans-Jürgen / Lerch, Marika (Hrsg.), Theorien der europäischen Integration. Wiesbaden: VS-Verlag, S. 399-423.

Hengsbach, Friedhelm (2001): Solidarität im Sturzflug? Eine sozialethische Reflexion. In: WSIMitteilungen, H. 8, S. 471-477.

Henke, Klaus-Dirk (2005): Wie lassen sich Gemeinwohl und Wettbewerb in der Krankenversicherung miteinander verbinden? - Eine nationale und europaweite Herausforderung. In: Kirchhof, Paul (Hrsg.): Gemeinwohl und Wettbewerb. Heidelberg: C.F. Müller, S. 129-146.

Hervey, Tamara K. (2002). The Legal Basis of European Community Public Health Policy. In: McKee, Martin / Mossialos, Elias / Baeten, Rita (eds.). The Impact of EU Law on Health Care Systems. Brussels: Peter Lang, 23-55.

Jacobs, Francis (2003): Schlussanträge des Generalanwalts Francis Jacobs in den verbundenen Rechtssachen C-264/01, C-306/01, C-354/01 und C-355/01. EuGH Pressemitteilung Nr. 44/03 vom 22.05.2003.

Kingreen, Thorsten (2007): Europarechtliche Implikationen des Entwurfs eines Gesetzes zur Stärkung des Wettbewerbs in der Gesetzlichen Krankenversicherung (GKV-

Wettbewerbsstärkungsgesetz - GKV-WSG). Rechtsgutachten für den Deutschen Gewerkschaftsbund und die Hans Böckler-Stiftung. http://www.boeckler.de/pdf_fof/S-2006-922-4-1.pdf (Zugriff am 15.01.08).

Klammer, Ute (1998): Gerechtigkeit in der Gesundheitsversorgung - von der Verfehlung des Ziels zur Auflösung der Zielvorstellung? In: Blasche, Siegfried / Döring, Dieter (Hrsg.): Sozialpolitik und Gerechtigkeit. Frankfurt/New York: Campus, S. 258-313.

Kommission der Europäischen Gemeinschaften (2001a): Europäisches Regieren. Ein Weißbuch. KOM(2001)428 endgültig. Brüssel, den 25.07.2001.

Kommission der Europäischen Gemeinschaften (2001b): Mitteilung der Kommission an den Rat, das Europäische Parlament, den Wirtschafts- und Sozialausschuss und den Ausschuss der Regionen. Die Zukunft des Gesundheitswesens und der Altenpflege: Zugänglichkeit, Qualität und langfristige Finanzierbarkeit sichern. KOM(2001) 723 endgültig, Brüssel, den 05.12.2001.

Kommission der Europäischen Gemeinschaften (2005): Mitteilung der Kommission. Sozialpolitische Agenda. KOM(2005) 33 endgültig. Brüssel, den 09.02.2005.

Lamping, Wolfram (2007): Die Kunst des Bohrens dicker Bretter. Die Europäische Kommission und die Europäisierung von Gesundheitspolitik. In: Fischer, Robert, Karrass, Anne, Kröger, Sandra (Hrsg.). Die Europäische Kommission und die Zukunft der EU. Ideenfabrik zwischen europäischem Auftrag und nationalen Interessen. Opladen/Farmington Hills: Budrich. 
Leibfried, Stephan (2006): Europäische Sozialpolitik - Richtern und Märkten überlassen? WSIMitteilungen 10/2006, 523-531.

Manow, Philipp / Schäfer, Armin / Zorn, Hendrik (2006), Europäische Sozialpolitik und Europas parteipolitisches Gravitationszentrum in den Jahren 1957-2003, in: Zeitschrift für internationale Beziehungen, 13. Jg. (2006), H. 1, S. 75-107.

Marshall, Thomas H. (1992): Bürgerrechte und soziale Klassen. Frankfurt/New York: Campus.

Permanand, Govin (2006): EU pharmaceutical regulation. The politics of policy-making. Manchester: University Press.

Rat der Europäischen Union (2006): Schlussfolgerungen des Rates zum Thema "Gemeinsame Werte und Prinzipien in den Europäischen Union-Gesundheitssystemen". In: Amtsblatt der Europäischen Union C 146 vom 22.06.2006, S. 1-3.

Scharpf, Fritz W. (2000), Notes Toward a Theory of Multilevel Governing in Europe. MPIfG Discussion Paper 00/5, Köln.

Scharpf, Fritz W. (2002): The European Social Model: Coping with the Challenges of Diversity. Journal of Common Market Studies, Vol. 40, No. 4, 645-70.

Scharpf, Fritz W. (2003), Politische Optionen im vollendeten Binnenmarkt, in: Jachtenfuchs, Markus / Kohler-Koch, Beate (Hrsg.), Europäische Integration. 2. Aufl. Opladen: Leske + Budrich, S. 219-253.

Schmucker, Rolf (2003): Europäischer Binnenmarkt und nationale Gesundheitspolitik. Zu den Auswirkungen der „vier Freiheiten“ auf die Gesundheitssysteme der Mitgliedsländer. In: Jahrbuch für Kritische Medizin 38. Hamburg: Argument Verlag, S. 107-120.

Schneider, Markus (2002): Gesundheitssystemforschung und Gesundheitsstatistik in der Europäischen Union. Stand und Perspektiven im Hinblick auf die "offene Methode der Koordinierung“. In: Gesundheit und Gesellschaft Wissenschaft 2/2002, S. 15-21.

Schneider, Markus (et.al.)(2007): Indikatoren der OMK im Gesundheitswesen und der Langzeitpflege. Gutachten für das Bundesministerium für Gesundheit. Augsburg: Basys.

Schreiber, Arnold (2005): Die Offene Methode der Koordinierung im Gesundheitswesen und zur aktuellen Situation. In: Sozialer Fortschritt 5-6/2005, S. 149-152.

Steinmeyer, Heinz-Dietrich (1999): Hat das Territorialitätsprinzip in der GKV noch Zukunft? In: Die Krankenversicherung, 10/1999, S. 288-291.

Tomuschat, Christian (2004): Die völkerrechtlichen Grundlagen der Zivilgesellschaft: Internationale Abkommen als Garanten internationaler Solidarität? In: Beckert u.a. (Hrsg.), S. 135-147. 
In der Reihe

Diskussionspapiere des Instituts für Medizinische Soziologie sind bisher erschienen:

2005 - 1 Rolf Schmucker: Die deutsche Arzneimittelzulassung im europäischen Wettbewerb Schutzgebühr 1,50 EUR

2006-1 Kai Mosebach: Patienten auf dem Weg zur Macht? Ergebnisse rot-grüner Gesundheitspolitik Schutzgebühr 2,50 EUR

2007-1 Thomas Gerlinger, Kai Mosebach, Rolf Schmucker: Wettbewerbssteuerung in der Gesundheitspolitik : Die Auswirkungen des GKV-WSG auf das Akteurshandeln im Gesundheitswesen Schutzgebühr 1,50 EUR

2007-2 Thomas Gerlinger: Soziale Ungleichheit von Gesundheitschancen: Anmerkungen zum Beitrag der Gesundheitspolitik Schutzgebühr 2,00 EUR

2008-1 Katharina Böhm: Politische Steuerung des Gesundheitswesens: Die Rolle von Korporatismus und Wettbewerb im Krankenhaussektor

Schutzgebühr 5,00 EUR

2008-2 Rolf Schmucker: Solidarität in der europäisierten Gesundheitspolitik?: Zum Verhältnis von Wettbewerb und Solidarität im europäischen Binnenmarktprojekt

Schutzgebühr 2,00 EUR

Die Diskussionspapiere sind gegen eine Schutzgebühr erhältlich bei:

Fachbereich Medizin der Johann Wolfgang Goethe-Universität Institut für Medizinische Soziologie Theodor-Stern-Kai 7, 60590 Frankfurt a. M.

Tel.: (0 69) 6301-76 10 / Fax: (0 69) 63 01-66 21 